\title{
Chronic effects of air pollution
}

\section{J G Ayres}

\section{Different approaches and different answers?}

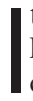
is now generally accepted that the health of many people is affected by exposure to air pollution on a day to day basis. The effects occur at varying levels of severity from mortality through hospital admissions to less serious morbidity such as increased use of inhalers in asthma. The quantification report ${ }^{1}$ of the United Kingdom Department of Health estimated that particles contribute to around 8500 deaths a year in the United Kingdom when considering the information available on these day to day effects. But there is evidence that exposure over time to polluted air can cause chronic effects either by initiating disease in otherwise healthy people or by enhancing the long term deterioration of a person's disease, ${ }^{2}$ and separating the two, if possible, is important for determination of the effects on public health.

Important evidence about chronic effects comes from three cohort studies (the Six Cities study, the American Cancer Society study ${ }^{4}$ and the Seventh Day Adventist study ${ }^{5}$ ) but cohort studies are expensive and take time to produce answers. As a result they are much treasured and considerable use has been made of the effect size coefficients from these studies to estimate the overall health impacts of air pollution in other countries, in particular as a step towards costing these impacts. But they have their shortcomings. For example, inadequate allowance may have been made for certain confounders such as socioeconomic factors, assessment of disease severity cannot be undertaken as they are based on routinely collected data and they are unable to take account of the effect of early life exposure to pollutants later in life. Along with population demographic and cultural differences and variations in qualitative aspects of the pollutant mix, these weaknesses may mean that extrapolating the findings of these studies, at least in quantitative terms, to other countries may be unwise.

Up to now the approach used in these American cohort studies has been the best available option. However, an alternative approach which could provide evidence on chronic effects using preexisting information would be very attractive if methodologically sound. Lipfert and Morris ${ }^{6}$ ( see pl56) used cross sectional regression analysis of the entire population of the United States (except
Alaska) based on mortality and air quality data but incorporating where available alternative datasets for potential confounders such as socioeconomic factors, lifestyle influences, and diet over nearly 40 years.

Is this a valid approach? The use of other datasets should improve accuracy, even though in some cases in this analysis the dataset used remains imperfect. For instance, using average traffic flows for a given county while underestimating exposure for part of the population and overestimating for some (there is reasonable evidence to show that health impacts are greater for those living within 100-200 yards (roughly 100-200 $\mathrm{m}$ ) of a main road) does provide a better estimate of exposure, particularly as there has been no attempt in the previous cohort studies to consider this directly. In common with the cohort studies, this approach cannot assess the subjects themselves, their diseases, and their severity; for large populations this is effectively impossible. Nevertheless, this remains an important missing piece in the jigsaw particularly if trying to assess susceptible subpopulations. Overall, however, the approach of Lipfert and Morris is logical and has the great advantage of providing answers quickly.

The analysis by Lipfert and Morris produces results which add to the information from the cohort studies in four main aspects: the diminishing effect of air pollution on mortality over time; the effect of age; geographical variation; and the possibility of identifying threshold levels for mortality.

Particularly in the earlier years of the period studied the effects of various pollutants was broadly greater in the 15-45 age group whereas in more recent times there was no association between mortality and exposure in the $\geqslant 85$ age group. These findings can be explained in terms of disease severity and susceptibility. The lack of association in the $\geqslant 85$ age group is not necessarily surprising. Those who have lived to be 85 are tough and it is likely that this age group could be considered a survivor populationthose susceptible to the adverse effects of air pollution may well have not survived to this age. The finding in the younger groups, especially earlier in the study period, is less easy to understand. Deaths in this age group, once trauma and AIDS have been removed, are relatively uncommon and could imply a very particular group of people who may be critically susceptible to many triggering events of which air pollution is but one. This undue susceptibility cannot be identified without having more specific details at a personal level.

The authors report thresholds for mortality although the thresholds are very sensitive to use of different measures of the same pollutant at different times making interpretation difficult. The shape of the mortality curve for particles is curious with the nadir of effect not being at the lowest level of exposure but at a point somewhere around the average exposure for the overall population. So although a threshold can be considered perhaps to be present at a level higher than this, it is still difficult to explain the U shaped curve even accepting the relative paucity of data at the lower end of the exposure range. This is more likely to be due to residual confounding as it is difficult to envisage a potential protective mechanism for moderate exposure to particles.

If these effects were independent of geography one would expect the presence and size of any association to be robust to whatever combination of areas were considered, unless there were clear differences in exposures or populations which intuitively would have an effect. This is not the case in this analysis. So does this completely undermine the belief that air pollution has an effect on health or does this simply mean that qualitative aspects of particles are more important than the mass of particles inhaled? The lack of coherence of the pattern of effects by mass of pollutant in this study might also point to the qualitative aspects of particles being more important. If so, then these findings also confirm how unwise it is to extrapolate effect size coefficients from one area to another.

This is an important study begging several questions. Limiting the areas of interest in the analysis to those covered by the two main cohort studies did result in coefficients broadly similar to those from the original studies, but it means that the findings need to be validated with datasets from other countries. If the findings hold true, not only does this require a biological explanation, it also raises the intriguing possibility that, at least in the United States, air pollution is having a progressively less important effect on mortality as we enter the 21 st century.

Occup Environ Med 2002;59: 147-148

\section{Authors' affiliations}

J G Ayres, Department of Respiratory Medicine, Birmingham Heartlands and Solihull NHS Trust (Teaching), Bordesley Green East, Birmingham, West Midlands B9 5SS, UK: ayresj@heartsol.wmids.nhs.uk 


\section{REFERENCES}

1 Committee On Medical Effects of Air

Pollution, Department of Health.

Quantification of the health effects of air

pollution in the UK. London: The Stationary

Office, 1997

2 Brunekreek B. Air pollution and life expectancy: is there a relation? Occup Environ Med 1997;54:781-4.
3 Dockery DW, Pope CA III, Xu X, et al. An association between air pollution and mortality in six US cities. N Engl J Med 1993;329:1753-9.

4 Pope CA, Thun M, Namboodiri MM, et al. Particulate air pollution as a predictor of mortality in a prospective study of US adults. Am J Respir Crit Care Med 1995:151:669-74.

5 Abbey DE, Nishino N, McDonnell WF, et al. Long-term inhalable particles and other air pollutants related to mortality in non-smokers. Am J Respir Crit Care Med 1999;159:373-82.

6 F W Lipfert, S C Morris. Temporal and spatial relations between age specific mortality and ambient air quality in the United States: preliminary results for counties,

1960-97. Occup Environ Med

2002:59:156-74.

\section{OEM web submission and review system}

I am pleased to inform authors and reviewers of the new online submission and review system at OEM. Developed by Highwire Press (CA, USA), Bench>Press is a fully integrated electronic system which uses the internet to allow rapid and efficient submission of manuscripts. It also allows the peer review process to be conducted entirely online. The main aim is to speed up the frequently frustrating progress from submission to publication.

Authors can submit their manuscript in any standard word processing software. Standard graphic formats acceptaed include: .jpg, .tiff, .gif, eps, etc. (Please note: multi page powerpoint files are not accepted by the BMJ Publishing Group.) The text and graphic files are automatically converted to PDF for ease of distribution and reviewing purposes. Authors are asked to approve their submission before it formally enters the reviewing process. On approval, the submission is passed to the editor and/or reviewers via the web. All transactions are secure.

To access the system click on "SUBMIT YOUR MANUSCRIPT HERE" on the OEM homepage: http://www.occenvmed.com, or you can access the submission site directly at http://submitoem.bmjjournals.com.

We are very excited with this new development and would encourage authors and reviewers to use the system where possible. It really is simple to use and should greatly improve on the current peer review process. Full instructions can be found on Bench>Press http://submitoem.bmjjournals.com and OEM online at http://www.occenvmed.com. Please contact Natalie Davies, Project Manager, ndavies@bmjgroup.com.

Anne Cockcroft Editor, OEM 